\title{
聚甲基丙烯酸 $(N$-羊隹基琥珀酰亚胺)酯的簇聚诱导发光研究
}

\author{
宾金貫卫剑袁望章* 张永明* \\ (上海交通大学化学化工学院 上海市电气绝缘与热老化重点实验室 上海 200240)
}

\begin{abstract}
摘要 不含普通发光单元的非典型生色团发光化合物因其基础研究重要性和广泛的应用前景引起了人们的极大兴趣. 其中许多化合物还具有独特的聚集诱导发光(Aggregation-induced emission, AIE)特性. 然而其发光机理仍然存在争议. 在此前的研究中, 提出了簇聚诱导发光(clustering-triggered emission, CTE)机理, 即非典型生色团的簇聚和电子共享来 解释这些体系的发光和 AIE 现象. 为进一步验证这一假说, 设计合成了不含传统生色团的聚甲基丙烯酸 $(N$-差基琥珀酰 亚胺)酯(PNHSMA). 其稀溶液基本不发光, 但浓溶液, 纳米聚集体, 固体粉末均发射蓝光, 呈现出 AIE 性质。通过与其 单体甲基丙烯酸 $(N$-差基琥珀酰亚胺)酷(NHSMA)的发光行为对比及单体单晶结构解析, 利用 CTE 机理很好地解释了其 光物理行为.

关键词 聚甲基丙烯酸 $(N$-羟基琥珀酰亚胺)酯; 聚集诱导发光; 非典型生色团; 簇聚诱导发光; 电子云重叠
\end{abstract}

\section{Clustering-Triggered Emission of $\operatorname{Poly}(\boldsymbol{N}$-hydroxysuccinimide Methacrylate) \\ Bin, Xin Luo, Weijian Yuan, Wangzhang* Zhang, Yongming* \\ (School of Chemistry and Chemical Engineering, Shanghai Key Lab of Electrical Insulation and Thermal Aging, Shanghai Jiao Tong University, Shanghai 200240)}

\begin{abstract}
Nonconventional luminogens without classic aromatic or conjugated structures are attracting increasing interests owing to their fundamental importance and promising applications in diverse areas. Many of them even exhibit unique aggregation-induced emission (AIE) characteristics. The emission mechanism, however, remains under debate. Previously, we proposed the clustering-triggered emission (CTE) mechanism, namely the clustering of nonconventional chromophores and subsequent electron overlap to rationalize the emission behaviors of such luminogens. To further our understanding, herein, we designed and synthesized poly( $N$-hydroxysuccinimide methacrylate) (PNHSMA) without any aromatic structures, which was obtained by the radical polymerization of $N$-hydroxysuccinimide methacrylate (NHSMA) monomer in toluene at $65{ }^{\circ} \mathrm{C}$ utilizing azobisisobutyronitrile (AIBN) as initiator. And NHSMA was prepared through the elimination between $\mathrm{N}$-hydroxysuccinimide (NHS) and methacryloyl chloride in the presence of triethylamine $\left(\mathrm{Et}_{3} \mathrm{~N}\right)$. It is found that PNHSMA is virtually nonluminescent in dilute solutions $\left(\leqslant 0.4 \mathrm{mg} \cdot \mathrm{mL}^{-1}\right)$ even at $77 \mathrm{~K}$, but gets emissive in concentrated solutions (e.g. $40 \mathrm{mg} \cdot \mathrm{mL}^{-1}$ ) with photoluminescence (PL) maxima at 434 and $485 \mathrm{~nm}$ at room temperature. Moreover, its solid powders emit intense blue light with multiple PL peaks upon UV irradiation, indicating its AIE nature and the formation of varying emission species. Further PL measurement of PNHSMA in dimethylformide (DMF) and DMF/acetone (good solvent/nonsolvent) mixtures duly verifies its AIE feature. Meanwhile, NHSMA monomer shows similar emission behaviors to those of PNHSMA, demonstrating concentration enhanced emission and AIE characteristics. In light of above results, it is assumed that NHSMA and its polymeric counterpart PNHSMA may share the similar emission mechanism. Single crystal structure of NHSMA reveals the conjugation of imide group and $3 \mathrm{D}$ intermolecular interactions of $\mathrm{C}=\mathrm{O} \cdots \mathrm{C}=\mathrm{O}(\mathrm{n}-\pi, 3.072$ $\AA), \mathrm{C}=\mathrm{O} \cdots \mathrm{H}-\mathrm{C}(2.651,2.642 \AA)$ and $\mathrm{C}=\mathrm{O} \cdots \mathrm{C}-\mathrm{H}(3.099 \AA)$. The emission of PNHSMA and NHSMA in concentrated solutions and solid states is thus ascribed to the clustering of imide and ester groups, which results in electronic interactions. The overlap of $\pi$ and lone pair (n) electrons among $\mathrm{C}=\mathrm{O}, \mathrm{N}$ and $\mathrm{O}$ units, together with effective intermolecular interactions in the solid powders extend the conjugation and rigidify the molecular conformations, thus leading to boosted emissions. Such CTE mechanism might be well extended to other nonconventional systems and should be inspiring for the rational design of novel luminogens.
\end{abstract}

Keywords poly( $N$-hydroxysuccinimide methacrylate); aggregation-induced emission; nonconventional luminogens; clustering-triggered emission; electron cloud overlap

\footnotetext{
*E-mail: wzhyuan@sjtu.edu.cn; ymzsjtu@gmail.com

Received August 20, 2016; published October 8, 2016.

Supporting information (including CCDC 1500208 for NHSMA) for this article is available free of charge via the Internet at http://sioc-journal.cn. Project supported by the National Natural Science Foundation of China (No. 51473092) and the Shanghai Rising-Star Program (No. 15QA1402500). 项目受国家自然科学基金(No. 51473092)和上海市青年科技启明星计划(No. 15QA1402500)资助.
} 


\section{1 引言}

有机发光材料因其在药物缓释 ${ }^{[1]}$, 有机发光二极 管 ${ }^{[2]}$, 生物成像 ${ }^{[3]}$, 生物传感器 ${ }^{[4]}$, 光波导 ${ }^{[5]}$ 等方面的潜 在应用受到广泛关注. 传统发光材料多为具有芳环或芳 杂环的共轭结构 ${ }^{[6]}$. 然而, 近年来, 有一类化合物因其 独特结构和内源性发光特性而被大量报道, 与传统的发 光材料相比, 这类化合物仅含有酰胺基团 $(\mathrm{CONH})$, 酯 基 $(\mathrm{COO})$, 羰基 $(\mathrm{C}=\mathrm{O})$ 等传统概念中的助色团, 而不含 苯环, 咔唑, 噻吩等芳香共轭结构, 却能在高浓溶液, 纳米悬浮液或固体粉末和薄膜等状态下发射明显的可 见光 ${ }^{[720]}$. 这类非典型发光化合物, 不仅合成较传统发 光材料更为容易, 而且可通过简单修饰使其获得良好的 亲水性和生物相容性, 从而在生物传感和影像等方面具 有良好的应用前景. 其发光机理的研究吸引了众多研究 者的注意. 对机理的探索, 可加深人们对这些体系光物 理过程的认识, 开发出更多的新型发光材料, 还可能为 我们揭开生物体系自发光(autofluorescence)的秘密. 然 而, 目前人们对这些体系的认识尚处在初始阶段, 对机 理的看法不尽相同. 例如对超支化聚酰胺胺 ${ }^{[11]}$ 、聚醚酰

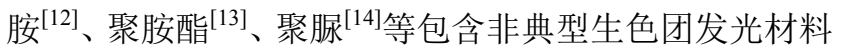
的机理解释尚存争议, 人们提出了氧化 ${ }^{[15]}$, 不饱和羟胺 的生成 ${ }^{[16]}$ 以及羰基的聚集 ${ }^{[17]}$ 等来解释. 在这些研究中, 人们较多地关注了外围取代基、化合物拓扑结构、环境 $\mathrm{pH}$ 、氧化等因素的影响, 而缺乏不同浓度, 不同聚集状 态, 不同温度条件下的系统研究, 从而导致研究结论片 面, 往往很快被后续研究证明并非如此 ${ }^{[7 a]}$.

在前期研究过程中, 我们观察到大米发光, 进一步 研究发现淀粉、纤维素、蛋白质等天然产物和生物分子 在稀溶液中不发光, 而在固态(或晶态)发光 ${ }^{[9]}$, 表现出 聚集诱导发光(AIE)特性 ${ }^{[18 ~ 20]}$. 根据这一类化合物的结 构和不同状态下的发光性质, 我们提出了富电子基团簇 聚形成实际发光生色团的机理: 簇中电子云相互重叠共 享, 使电子离域, 共轭扩展, 从而在构象适当刚硬化的 条件下受激发光. 这一机理也很好地解释了聚丙烯腈 $(\mathrm{PAN})$ 化合物在高浓溶液和固态的发光 ${ }^{[b]}$, 其 $\mathrm{C} \equiv \mathrm{N}$ 侧 基的簇聚使基团间孤对 $\mathrm{n}$ 电子和 $\pi$ 电子之间相互作用与 重叠, 这对发光而言至关重要. 我们将上述非典型生色 团化合物的发光称之为簇聚诱导发光 (clustering-triggered emission) ${ }^{[9 b]}$. 在最近的报道中, 人 们发现了很多具有内源性发光的非典型生色团化合 物 ${ }^{[\sim 17,21 \sim 23]}$. 其中, 我国学者在这一领域的研究尤为突 出, 取得了重要研究进展 ${ }^{[8 \sim 10,11 \mathrm{c} \sim 11 \mathrm{~g}, 16,17]}$. 许多学者也利 用基团簇聚来解释相应的体系 ${ }^{[8,9,21 ~ 24]}$, 如线形和超支

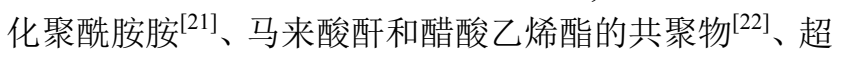
支化聚梄 ${ }^{[23]}$ 等.

基于前期工作和文献报道, 我们认为簇聚诱导发光 机理, 即非典型生色团的簇聚导致的电子云重叠能够较 合理地解释目前报道的体系, 并可能推广到其他体系.
为进一步验证该机理，我们设计合成了包含酰亚胺基团 和酯基的聚甲基丙烯酸 ( $N$ - 羟基琥珀酰亚胺) 酯 (PNHSMA), 研究了其溶液、纳米聚集体、固体粉末等 不同状态的发光性质，并与其单体 $N$-甲基丙烯酸琥珀 酰亚胺酯 (NHSMA) 的发光性质进行了比较, 通过对 NHSMA 的单晶结构分析提出了相应的机理解释.

\section{2 结果与讨论}

\section{1 聚合物的合成}

如图式 1 所示, 单体 NHSMA 由 $N$-差基琥珀酰亚胺 (NHS) 和甲基丙烯酰氯(MAC)在 $\mathrm{Et}_{3} \mathrm{~N}$ 存在条件下反应制 得, 其在甲苯溶剂中由 $\mathrm{AIBN}$ 作引发剂, 于 $65{ }^{\circ} \mathrm{C}$ 进一 步聚合得到最终产物 PNHSMA ${ }^{[25]}$. 核磁与单晶结构 (CCDC 1500208)解析进一步证实了目标单体和聚合物 的成功制备(图 S1, S2). 凝胶渗透色谱(GPC)结果显示 PNHSMA 的重均分子量为 $2.4 \times 10^{4} \mathrm{~g} \cdot \mathrm{mol}^{-1}$, 分子量分 布为 4.5 (图 S3).

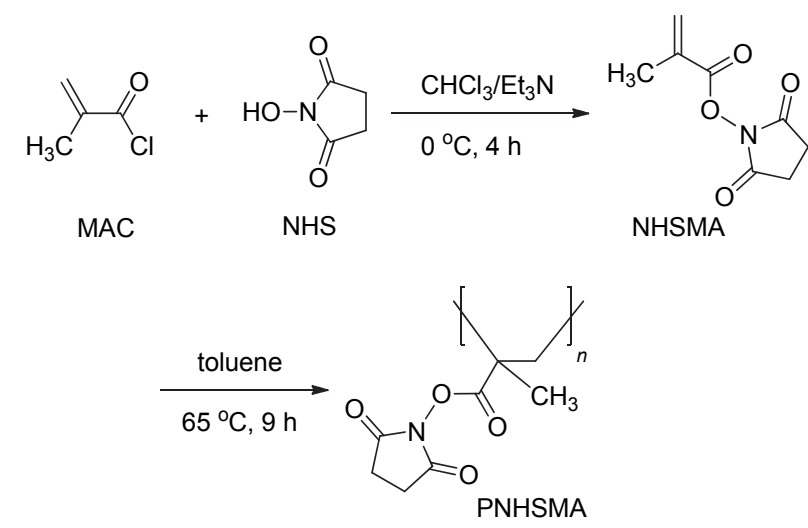

图式 1 聚合物 PNHSMA 的合成路线

Scheme 1 Synthetic route to PNHSMA

\subsection{PNHSMA 的发光性质}

在 $365 \mathrm{~nm}$ 紫外光辐照下, PNHSMA 白色固体粉末 发出明亮蓝光(图 1A，B). 如图 1C 所示, 其发射谱在 $345,365 \mathrm{~nm}$ 紫外光激发下, 在 433, 470, 485, 496, 540, $570,595 \mathrm{~nm}$ 等处具有发射(肩)峰, 这可能是 PNHSMA 固体具有多种不同的聚集状态，从而形成了多个发射种 造成的.

为进一步探索 PNHSMA 的发光机理, 配制了一系 列浓度的二甲基甲酰胺(DMF)溶液, 并测试了其吸收和 发射谱. 如图 2A 所示, PNHSMA 稀溶液不发光, 当浓度 增加至 $4 \mathrm{mg} \cdot \mathrm{mL}^{-1}$ 时, 仍无明显发光, 进一步增至 10 $\mathrm{mg} \cdot \mathrm{mL}^{-1}$, 开始观测到微弱发光. 随着溶液浓度的进一 步升高, 其发光强度逐步增强, 当浓度为 $40 \mathrm{mg} \cdot \mathrm{mL}^{-1}$ 时, 在 $365 \mathrm{~nm}$ 的紫外光辐照下可观测到明显的蓝光. 这 些结果说明 PNHSMA 溶液具有浓度增强发光性质, 这 与普通发光化合物的浓度猝灭效应正好相反. 同时, 稀 溶液不发光，而固体粉末发光则显示 PNHSMA 具有 

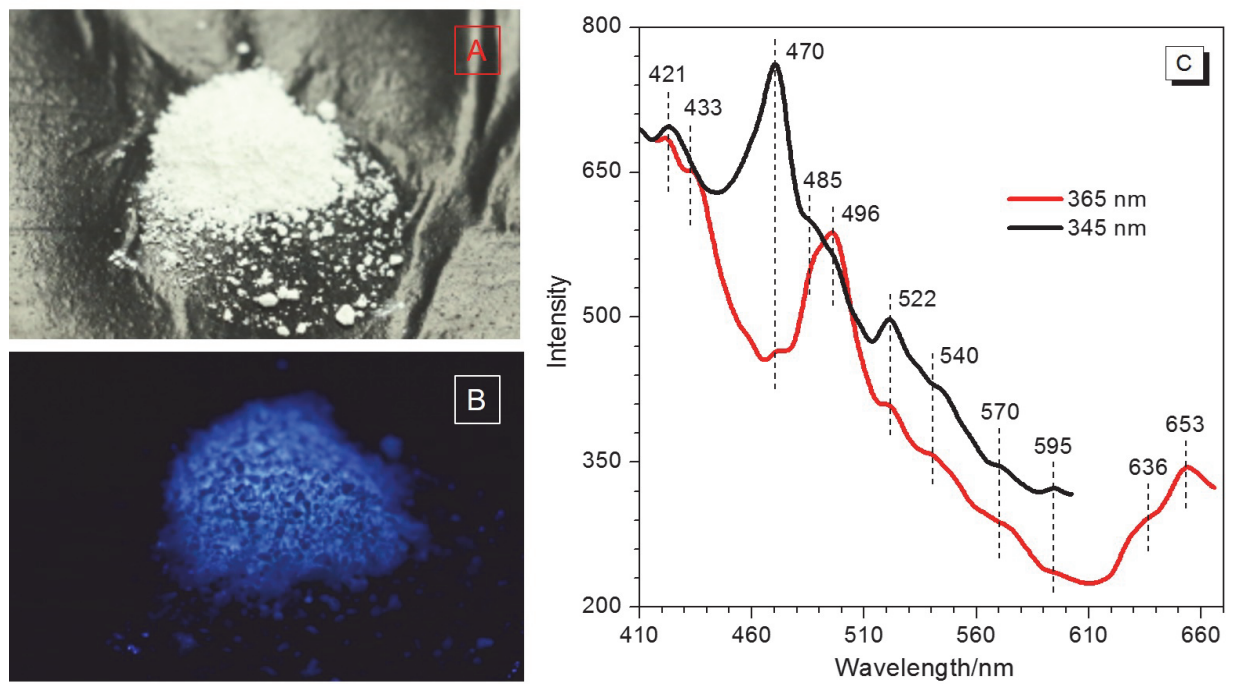

图 1 PNHSMA 固体粉末在(A)日光灯, (B) $365 \mathrm{~nm}$ 紫外灯下的照片. (C) PNHSMA 固体粉末在 345, $365 \mathrm{~nm}$ 紫外光激发下的发射谱

Figure 1 Photographs of PNHSMA solid powders taken under (A) room light and (B) $365 \mathrm{~nm}$ UV light. (C) Emission spectra of PNHSMA solid powders with excitation wavelengths of 345 and $365 \mathrm{~nm}$
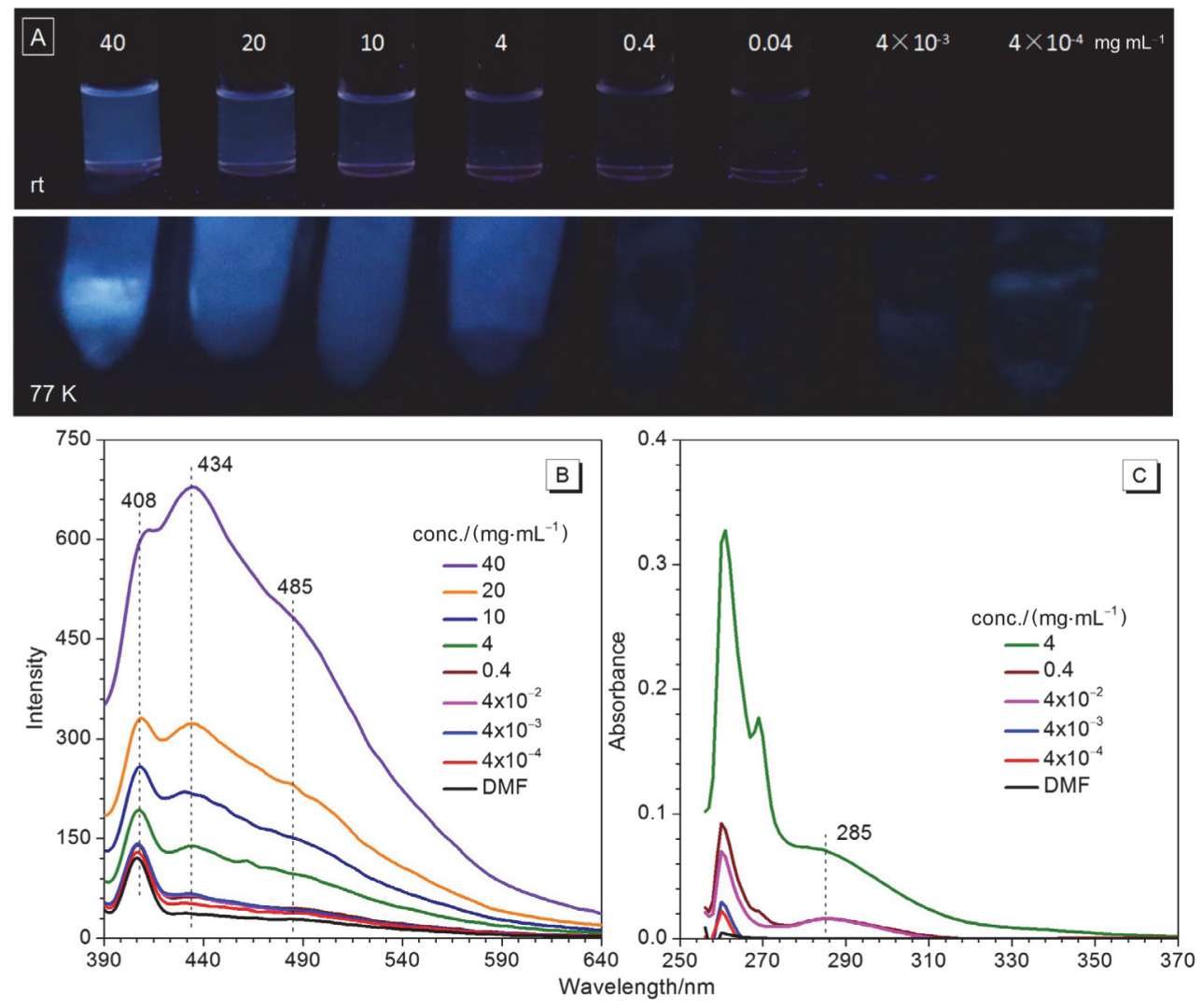

图 2 不同浓度 PNHSMA/DMF 溶液(A)在室温(上)和 $77 \mathrm{~K}$ (下)置于 $365 \mathrm{~nm}$ 紫外光辐照下的照片, (B)发射谱 $\left(\lambda_{\mathrm{ex}}=365 \mathrm{~nm}\right.$ )及 $(\mathrm{C})$ 吸收谱

Figure 2 (A) Photographs taken at room temperature (r.t., upper) and $77 \mathrm{~K}$ (lower) under $365 \mathrm{~nm}$ UV irradiation, (B) emission spectra $\left(\lambda_{\mathrm{ex}}=365 \mathrm{~nm}\right)$ and (C) absorption spectra of PNHSMA/DMF solutions at different concentrations

AIE 特性. 另外, 低温实验表明, 当溶液浓度 $\leqslant 0.4$ $\mathrm{mg} \cdot \mathrm{mL}^{-1}$ 时, 即便在 $77 \mathrm{~K}$ 的玻璃态也无法观测到明显的 可见光发射(图 2A), 说明单纯通过低温构象刚硬化来减 少分子运动也不能使 PNAMS 分子有效发光. 这一现象 与在传统发光化合物及典型芳香型 AIE 分子中的观察
都不相同，因此其发光机理也可能与这些材料不同. 而 当浓度 $\geqslant 4 \mathrm{mg} \cdot \mathrm{mL}^{-1}$ 时, 低温发射则比室温发射强, 这 是因为构象刚硬化使激子因振动, 转动造成的非辐射跃 迁减少造成的.

进一步测试了各溶液的发射谱, 发现低浓度溶液 
$\left(\leqslant 0.4 \mathrm{mg} \cdot \mathrm{mL}^{-1}\right)$ 的发射与纯 DMF 信号相差无几，当浓 度 $\geqslant 4 \mathrm{mg} \cdot \mathrm{mL}^{-1}$ 时, 发射随浓度增加而明显增强(图 2B). 在低浓度时, PNHSMA 溶液仅在 $408 \mathrm{~nm}$ 处有明显的发 射峰, 这主要来源于溶剂 DMF. 其高浓溶液则在 434, $485 \mathrm{~nm}$ 处出现发射(肩)峰, 与固体粉末的某些峰位相 近，可能形成了类似的聚集体. 图 $2 \mathrm{C}$ 吸收谱图显示，除 $285 \mathrm{~nm}$ 处吸收随浓度增加增强外, 当浓度为 $4 \mathrm{mg} \cdot \mathrm{mL}^{-1}$ 时, 在 $310 \mathrm{~nm}$ 以后区域也出现了新的吸收尾, 从而证实 了聚集体的形成. 利用积分球进一步测试了 $4 \times 10^{-4}, 4$ 及 $40 \mathrm{mg} \cdot \mathrm{mL}^{-1}$ 的 PNHSMA 溶液在室温条件下的绝对量 子效率，其数值分别为 $0.18 \%, 0.65 \%$ 及 $3.14 \%$ 。这一定 量结果进一步表明随着浓度的增加, PNHSMA 溶液经历 了由几乎不发光到发光的过程, 与肉眼观测结果一致.

PNHSMA 低浓度溶液, 无论在室温还是低温均不 发光, 而其高浓溶液和固体发光, 这可能是因为在低浓 度时聚合物分子链之间距离较远, 分子链较为伸展, 链 上侧基相互较为独立，没有形成有效聚集，因此无法被 有效激发. 随着浓度增加, 分子链之间的距离减少, 分 子链坡缩并形成链间缠结, 从而侧链能够形成一定程度
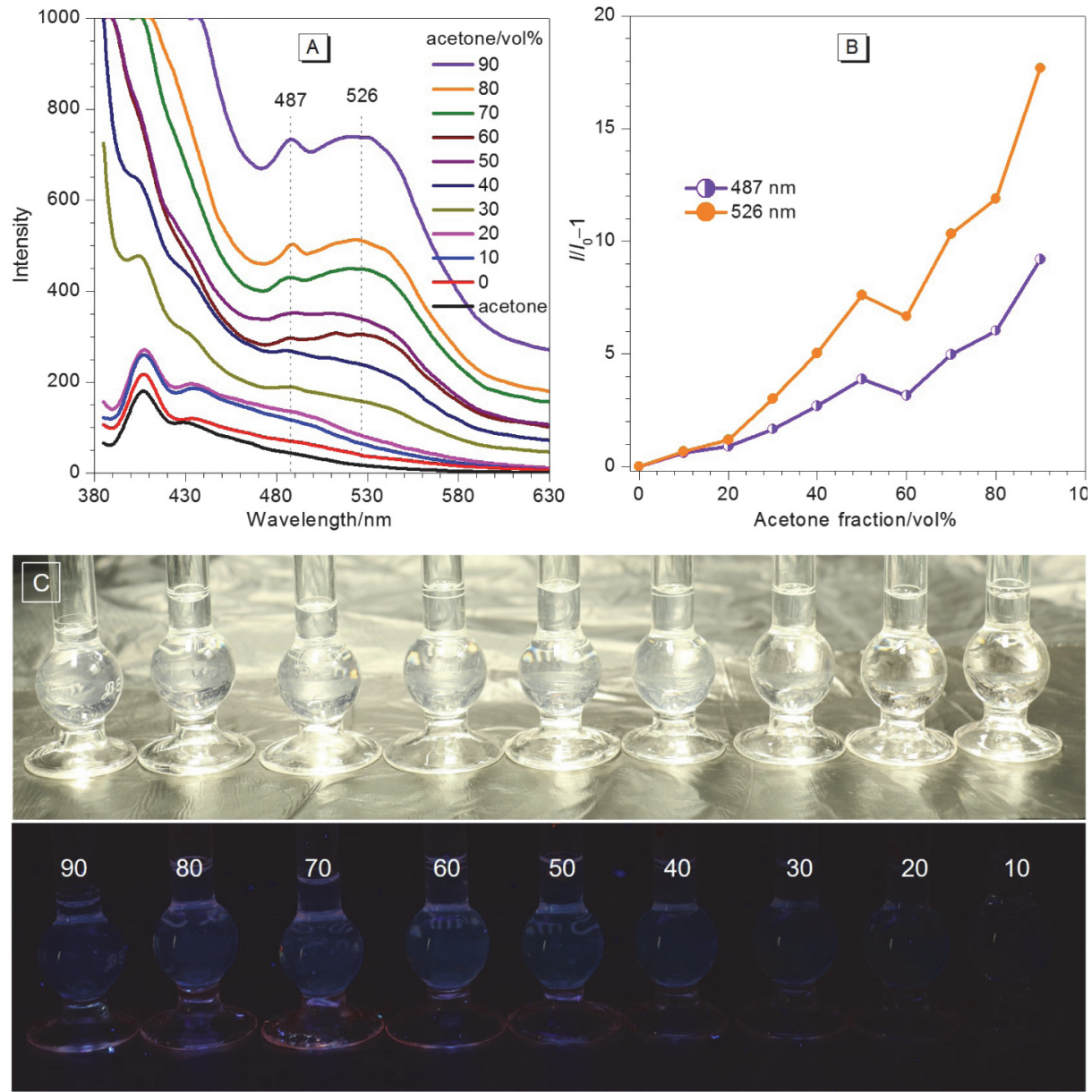

图 3 PNHSMA $\left(0.4 \mathrm{mg} \cdot \mathrm{mL}^{-1}\right)$ 在 DMF 及 $\mathrm{DMF} /$ 丙酮混合体系中的 $(\mathrm{A})$ 发射谱图, (B)峰位 $(487,526 \mathrm{~nm})$ 强度在不同丙酮含量下的净增比 $\left(I / I_{0}-1\right)$ 及 (C)在日光(上)和 $365 \mathrm{~nm}$ 紫外光(下)照射下的照片. 丙酮溶剂的发射也在(A)中给出

Figure 3 (A) PL spectra, (B) net increase of peak emission intensity $\left(I / I_{0}-1\right)$ at 487 and $526 \mathrm{~nm}$ and (C) photographs taken under room light (upper) and $365 \mathrm{~nm}$ UV light (lower) of PNHSMA in DMF and DMF/acetone mixtures $\left(0.4 \mathrm{mg} \cdot \mathrm{mL}^{-1}\right)$ with different acetone fractions. Emission spectrum of distilled acetone is also given in (A) 
$526 \mathrm{~nm}$ 处的发射最高增加了 9 和 18 倍(图 3B). 图 3C 给出了 PNHSMA 在 DMF 及 DMF/丙酮混合体系中的照 片, 尽管聚集后总体发光亮度较弱, 但从中仍可清晰地 看到其由不发光到发光的 AIE 过程.

\subsection{NHSMA 的发光性质}

为进一步阐明 PNHSMA 的发光和 AIE 机理, 对 NHSMA 单体在溶液及固态的发光性质进行了研究. 由 图 4 可知, 无论是其 DMF 溶液还是固体粉末, NHSMA 单体都表现出同其聚合物类似的发射行为, 呈现出浓度 增强发光和 AIE 现象. 高浓度溶液条件下, 除 $408 \mathrm{~nm}$ 处 的溶剂峰, NHSMA 在 432, $488 \mathrm{~nm}$ 处具有与聚合物相似 的发射峰. 同时, 其固体粉末在 443, 486, 530, 543, 575 $\mathrm{nm}$ 等处呈现发射峰位. 溶液和固体的多重发射峰也表 明多个聚集发光中心的存在. 对比单体和聚合物的 $\mathrm{DMF}$ 溶液 $\left(40 \mathrm{mg} \cdot \mathrm{mL}^{-1}\right)$ 发射谱, 发现两者在 $365 \mathrm{~nm}$ 紫 外光激发下基本相同(图 S4), 这进一步说明发光来源于 侧基, 且单体中双键对发射的影响较小. 上述实验结果 也说明单体与聚合物的发射机理大致相同.

由于 NHSMA 单体及其聚合物的发射行为和机理 类似, 我们研究了 NHSMA 的单晶结构. 如图 5 所示, $\mathrm{C} 1-\mathrm{N} 1, \mathrm{C} 4-\mathrm{N} 1$ 的键长分别为 1.377 和 $1.384 \AA$, 均小 于普通 $\mathrm{C}-\mathrm{N}$ 单键的键长, 且其五元环近乎平面结构, 这些说明 $\mathrm{N}$ 原子与两个 $\mathrm{C}=\mathrm{O}$ 形成了共轭结构. NHSMA
上酰亚胺基团与 $\mathrm{C}=\mathrm{O}$ 通过 $\mathrm{O}$ 原子相连，电子云相互作 用, 形成一定共轭，使整个共轭扩展。但这样的共轭还 较难被激发，也不足以产生可见光，因此单体和聚合物 稀溶液即便在 $77 \mathrm{~K}$ 也无法产生有效发光. 当分子聚集 时, 双键 $\pi$ 电子, $\mathrm{N}, \mathrm{O}$ 上的孤对电子相互之间可能出现 重叠, 从而发生电子离域. 这不仅使化合物构象刚性增 加, 更重要的是使分子共轭进一步扩展, 从而化合物容 易被激发而产生可见光发射. 同时, 分子中存在着 $\mathrm{C}=$ $\mathrm{O} \cdots \mathrm{C}=\mathrm{O}(\mathrm{n}-\pi, 3.072 \AA), \mathrm{C}=\mathrm{O} \cdots \mathrm{H}-\mathrm{C}(2.651,2.642 \AA)$ 及 $\mathrm{C}=\mathrm{O} \cdots \mathrm{C}-\mathrm{H}(3.099 \AA)$ 等分子间相互作用(图 5), 其 形成 3D网络, 使分子构象更加刚硬化, 可有效地减少 非辐射失活而增强光发射.

PNHSMA 由单体 NHSMA 聚合而成, 其发光机理 也可用上述机理合理解释. 在低浓度下, 高分子链在溶 液中呈舒展状态，分子间距离较大，较难形成聚集，共 轭长度小，难以被有效激发。而在高浓度下，高分子链 发生聚集甚至缠结，从而使侧基产生簇聚. 在此状态下, $\mathrm{O}$ 和 $\mathrm{N}$ 原子上的孤对电子所占据的 $\mathrm{p}$ 轨道相互发生交 叠, $\mathrm{C}=\mathrm{O}$ 的 $\pi$ 轨道可能会与其他 $\mathrm{C}=\mathrm{O} \pi$ 轨道通过偶极 偶极相互作用发生交叠, 孤对 $\mathrm{n}$ 电子也可与共轭 $\pi$ 产生 $\mathrm{n}-\boldsymbol{\pi}$ 相互作用. 这些非典型生色团的簇聚最终使电子云 重叠, 离域扩展, 分子构象刚性增大, 易于被激发而产 生可见光发射. 同时, 聚集态分子间相互作用以及固态
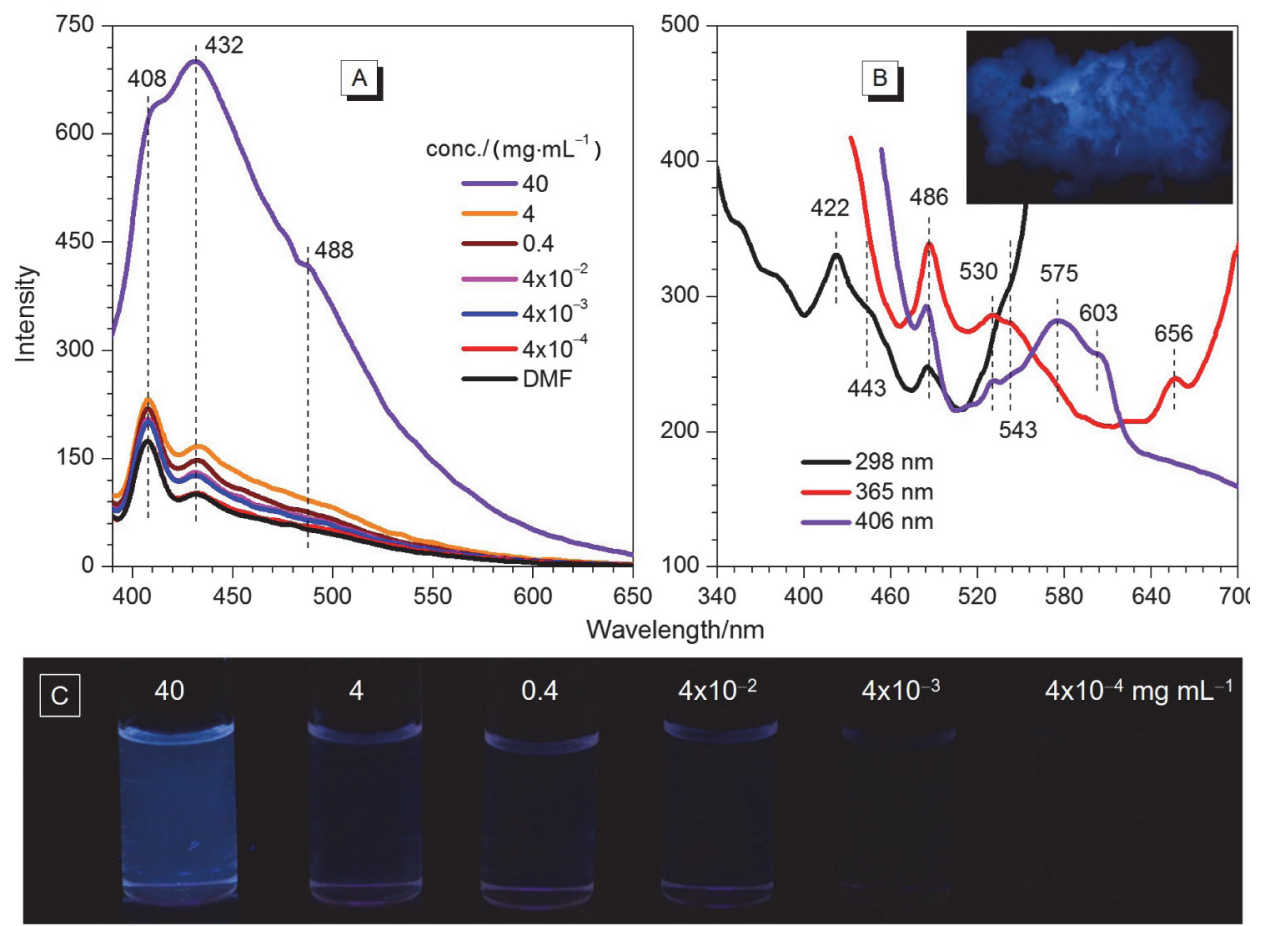

图 4 (A)不同浓度 NHSMA/DMF 溶液的发射谱 $\left(\lambda_{\mathrm{ex}}=365 \mathrm{~nm}\right.$ ). (B) NHSMA 固体粉末在 298, 365 及 $406 \mathrm{~nm}$ 紫外光辐照下的发射谱及其在 $365 \mathrm{~nm}$ 紫外光照下的照片. (C)不同浓度 NHSMA/DMF 溶液在 $365 \mathrm{~nm}$ 紫外光辐照下的照片

Figure 4 (A) Emission spectra of NHSMA/DMF solutions at different concentrations $\left(\lambda_{\mathrm{ex}}=365 \mathrm{~nm}\right)$. (B) Emission spectra of NHSMA solid powders with $\lambda_{\text {ex }}$ of 298,365 , and $406 \mathrm{~nm}$ and its photograph taken under $365 \mathrm{~nm}$ UV light. (C) Photograph of NHSMA/DMF solutions at different concentrations taken under $365 \mathrm{~nm}$ UV light 


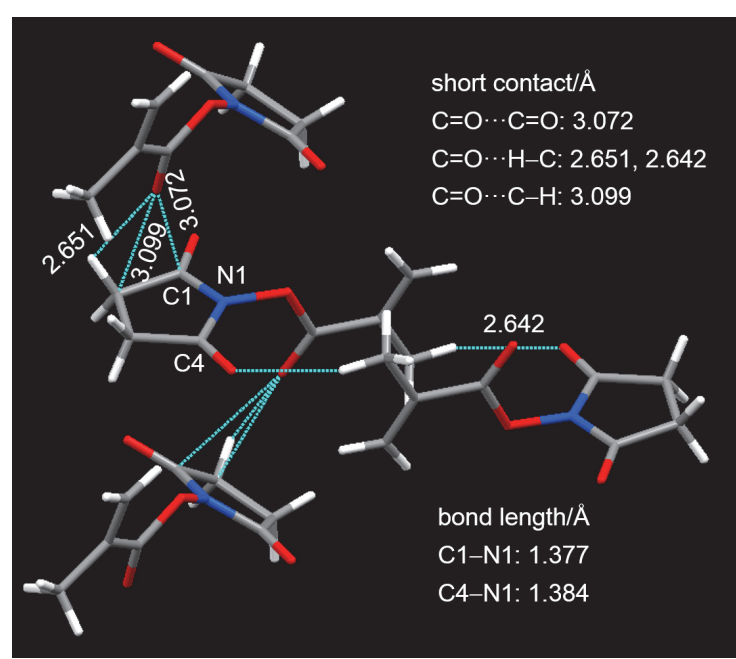

图 5 NHSMA 晶体结构及其局部分子排列与相互作用

Figure 5 Crystal structure of NHSMA and its fragmental packing with intermolecular interactions around one molecule indicated

聚合物链缠结等使构象刚硬化, 减少了非辐射跃迁的产 生, 从而进一步促进光发射.

\section{3 结论}

设计合成了不含苯环、噻吩、茄、咔唑等任何芳香 共轭单元的 PNHSMA, 其稀溶液不发光, 浓溶液和固体 粉末发射蓝光, 表现出 AIE 活性. 通过低温发光实验及 单体发光性质的对比和单晶结构解析, 利用非典型生色 团簇聚诱导发光机理很好地解释了单体及 PNHSMA 的 发光及 AIE 特性. 在低浓溶液中, 分子或侧基彼此分开, 难以被激发, 即便在 $77 \mathrm{~K}$ 也不发光. 在高浓溶液和固 态, 酰亚胺酯基团簇聚, 簇中 $\mathrm{C}=\mathrm{O}, \mathrm{N}, \mathrm{O}$ 单元的 $\pi$ 电子 和 $\mathrm{n}$ 电子相互重叠, 使得化合物有效共轭增加, 形成实 际的发光中心，同时分子刚性由于电子共享和分子间相 互作用而增大, 从而能够被激发并产生有效发射. 这一 簇聚诱导发光机理对后期新型非典型生色团发光化合 物的合理设计具有重要指导意义, 并有望进一步揭示生 物自发光背后的秘密.

\section{References}

[1] (a) Qiu, F.; Wang, D.; Zhu, Q.; Zhu, L.; Tong, G.; Lu, Y.; Yan, D.; Zhu, X. Biomacromolecules 2014, 15, 1355. (b) Kang, B.; Afifi, M. M.; Austin, L. A.; El-Sayed, M. A. ACS Nano 2013, 7, 7420.

[2] (a) Tao, Y.; Yang, C.; Qin, J. Chem. Soc. Rev. 2011, 40, 2943. (b) Wang, Q.; Ding, J.; Ma, D.; Cheng, Y.; Wang, L.; Jing, X.; Wang, F. Adv. Funct. Mater. 2009, 19, 84. (c) Zhang, Q.; Zhou, Q.; Cheng, Y.; Wang, L.; Ma, D.; Jing, X.; Wang, F. Adv. Mater. 2004, 16, 432. (d) Yang, J.; Li, L.; Yu, Y.; Ren, Z.; Peng, Q.; Ye, S.; Li, Q.; Li, Z. Mater. Chem. Front. 2017, 1, DOI: 10.1039/C6QM00014B.

[3] (a) Bai, L.; Li, W.; Chen, J.; Bo, F.; Gao, B.; Liu, H.; Li, J.; Wu, Y.; Ba, X. Macromol. Rapid Commun. 2013, 34, 539. (b) Wu, Y. X.; Li, J. B.; Liang, L. H.; Lu, D. Q.; Zhang, J.; Mao, G. J.; Zhou, L. Y.; Zhang, X. B.; Tan, W.; Shen, G. L.; Yu, R. Q. Chem. Commun. 2014, 50, 2040. (c) Shi, H.; Sun, H.; Yang, H.; Liu, S.; Jenkins, G.; Feng, W.; Li, F.; Zhao, Q.; Liu, B.; Huang, W. Adv. Funct. Mater. 2013, 23, 3268. (d) Chen, M.; Yin, M. Prog. Polym. Sci. 2014, 39, 365.

[4] (a) Song, Z.; Hong, Y.; Kwok, R. T. K.; Lam, J. W. Y.; Liu, B.; Tang, B. Z. J. Mater. Chem. B 2014, 2, 1717. (b) Zhang, W.; Xu, L.; Qin,
J.; Yang, C. Macromol. Rapid Commun. 2013, 34, 442. (c) Wang, M.; Zhang, G.; Zhang, D.; Zhu, D.; Tang, B. Z. J. Mater. Chem. 2010, 20, 185.

[5] (a) Zhao, Y. S.; Xu, J.; Peng, A.; Fu, H.; Ma, Y.; Jiang, L.; Yao, J. Angew. Chem. 2008, 120, 7411. (b) Gu, X.; Yao, J.; Zhang, G.; Yan, Y.; Zhang, C.; Peng, Q.; Liao, Q.; Wu, Y.; Xu, Z.; Zhao, Y.; Fu, H.; Zhang, D. Adv. Funct. Mater. 2012, 22, 4862.

[6] (a) Wang, K.; Zhang, H.; Chen, S.; Yang, G.; Zhang, J.; Tian, W.; Su, Z.; Wang, Y. Adv. Mater. 2014, 26, 6168. (b) Liu, D.; Duan, Y.-H. Chin. Chem. Lett. 2013, 24, 809. (c) Yuan, W. Z.; Lu, P.; Chen, S.; Lam, J. W. Y.; Wang, Z.; Liu, Y.; Kwok, H. S.; Ma, Y.; Tang, B. Z. Adv. Mater. 2010, 22, 2159. (d) Yuan, W. Z.; Gong, Y.; Chen, S.; Shen, X. Y.; Lam, J. W. Y.; Lu, P.; Lu, Y.; Wang, Z.; Hu, R.; Xie, N.; Kwok, H. S.; Zhang, Y.; Sun, J. Z.; Tang, B. Z. Chem. Mater. 2012, 24, 1518. (e) Ning, Z.; Chen, Z.; Zhang, Q.; Yan, Y.; Qian, S.; Cao, Y.; Tian, H. Adv. Funct. Mater. 2007, 17, 3799. (f) Wang, C.-R.; Gong, Y.-Y.; Yuan, W.-Z.; Zhang, Y.-M. Chin. Chem. Lett. 2016, 27, 1184.

[7] (a) Huang, T.; Wang, Z.; Qin, A.; Sun, J. Z.; Tang, B. Z. Acta Chim. Sinica 2013, 71, 979 (in Chinese). (黄田, 汪昭昒, 秦安军, 孙景 志, 唐本忠, 化学学报, 2013, 71, 979.) (b) Zhu, S.; Song, Y.; Shao, J.; Zhao, X.; Yang, B. Angew. Chem., Int. Ed. 2015, 54, 14626. (c) Pastor-Pérez, L.; Chen, Y.; Shen, Z.; Lahoz, A.; Stiriba, S.-E. Macromol. Rapid Commun. 2007, 28, 1404.

[8] (a) Yu, W.; Wu, Y.; Chen, J.; Duan, X. Y.; Jiang, X.-F.; Qiu, X.; Li, Y. RSC Adv. 2016, 6, 51257. (b) Yu, W.; Wang, Z.; Yang, D.; Ouyang, X.; Qiu, X.; Li, Y. RSC Adv. 2016, 6, 47632.

[9] (a) Gong, Y. Y.; Tan, Y. Q.; Mei, J.; Zhang, Y. R.; Yuan, W. Z.; Zhang, Y. M.; Sun, J. Z.; Tang, B. Z. Sci. China Chem. 2013, 56, 1178. (b) Zhou, Q.; Cao, B.; Zhu, C.; Xu, S.; Gong, Y.; Yuan, W. Z.; Zhang, Y. Small 2016, 12, DOI: 10.1002/smll.201601545.

[10] (a) Zhu, S.; Zhang, J.; Wang, L.; Song, Y.; Zhang, G.; Wang, H.; Yang, B. Chem. Commun. 2012, 48, 10889. (b) Niu, S.; Yan, H.; Chen, Z.; Li, S.; Xu, P.; Zhi, X. Polym. Chem. 2016, 7, 3747. (c) Wang, H.-X.; Yang, Z.; Liu, Z.-G.; Wan, J.-Y.; Xiao, J.; Zhang, H.-L. Chem. Eur. J. 2016, 22, 8096. (d) Liu, S. G.; Li, N.; Ling, Y.; Kang, B. H.; Geng, S.; Li, N. B.; Luo, H. Q. Langmuir 2016, 32, 1881.

[11] (a) Crosby, G. A.; Demas, J. N. J. Phys. Chem. 1971, 75, 991. (b) Lee, W. I.; Bae, Y.; Bard, A. J. J. Am. Chem. Soc. 2004, 126, 8358. (c) Cao, L.; Yang, W.; Wang, C.; Fu, S. J. Macromol. Sci. Part A. 2007, 44, 417. (d) Yang, W.; Pan, C.-Y. Macromol. Rapid Commun. 2009, 30, 2096. (e) You, Y. Z.; Yu, Z. Q.; Cui, M. M.; Hong, C. Y. Angew. Chem. Int. Ed. 2010, 49, 1099. (f) Wang, D.; Yu, Z.-Q.; Hong, C.-Y.; You, Y.-Z. Eur. Polym. J. 2013, 49, 4189. (g) Yang, L.; Wang, L.; Cui, C.; Lei, J.; Zhang, J. Chem. Commun. 2016, 52, 6154.

[12] Lin, Y.; Gao, J.-W.; Liu, H.-W.; Li, Y.-S. Macromolecules 2009, 42, 3237.

[13] (a) Wu, D. C.; Liu, Y.; He, C. B.; Goh, S. H. Macromolecules 2005 , 38, 9906. (b) Sun, M.; Hong, C.-Y.; Pan, C.-Y. J. Am. Chem. Soc. 2012, 134, 20581.

[14] Restani, R. B.; Morgado, P. I.; Ribeiro, M. P.; Correia, I. J.; Aguiar-Ricardo, A.; Bonifácio, V. D. B. Angew. Chem. Int. Ed. 2012, 51,5162 .

[15] Wang, D.; Imae, T. J. Am. Chem. Soc. 2004, 126, 13204.

[16] Lin, S. Y.; Wu, T. H.; Jao, Y. C.; Liu, C. P.; Lo, L. W.; Yang, C. S. Chem. Eur. J. 2011, 17, 7158.

[17] Lu, H.; Feng, L.; Li, S.; Zhang, J.; Lu, H.; Feng, S. Macromolecules 2015, 48, 476 .

[18] (a) Mei, J.; Leung, N. L. C.; Kwok, R. T. K.; Lam, J. W. Y.; Tang, B. Z. Chem. Rev. 2015, 115, 11718. (b) Hong, Y.; Lam, J. W. Y.; Tang, B. Z. Chem. Soc. Rev. 2011, 40, 5361. (c) Luo, J.; Xie, Z.; Lam, J. W. Y.; Cheng, L.; Chen, H.; Qiu, C.; Kwok, H. S.; Zhan, X.; Liu, Y.; Zhu, D.; Tang, B. Z. Chem. Commun. 2001, 1740. (d) Wang, J.; Mei, J.; Hu, R.; Sun, J. Z.; Qin, A.; Tang, B. Z. J. Am. Chem. Soc. 2012, 134, 9956. (e) Zhao, Z.; He, B.; Tang, B. Z. Chem. Sci. 2015, 6, 5347.

[19] (a) Li, C.; Tang, X.; Zhang, L.; Li, C.; Liu, Z.; Bo, Z.; Dong, Y. Q.; Tian, Y.-H.; Dong, Y.; Tang, B. Z. Adv. Opt. Mater. 2015, 3, 1184. (b) Zhao, Z.; Chen, T.; Jiang, S.; Liu, Z.; Fang, D.; Dong, Y. Q. J. Mater. Chem. C 2016, 4, 4800. (c) Feng, C.; Wang, K.; Xu, Y.; Liu, L.; Zou, B.; Lu, P. Chem. Commun. 2016, 52, 3836. (d) Yue, B.-L.; Xie, Z.-Q.; Lu, P.; Ma, Y.-G. Sci. Sinica Chim. 2013, 43, 1065 (in Chinese). (越柏玲, 解增旗, 路萍, 马於光, 中国科学: 化学, 2013, 43, 1065.) 
[20] (a) Xun, Z.-Q.; Tang, H.-Y.; Zeng, Y.; Chen, J.-P.; Yu, T.-J.; Zhang, X.-H.; Li, Y. Acta Chim. Sinica 2015, 73, 819 (in Chinese). (寻知庆, 唐海云, 曾毅, 陈金平, 于天君，张小辉，李嫕，化学学报，2015, 73, 819.) (b) Xia, Z.-Q.; Shao, A.-D.; Li, Q.; Zhu, S.-Q.; Zhu, W.-H. Acta Chim. Sinica 2016, 74, 351 (in Chinese). (夏志清, 邵安东, 李 强, 朱世琴, 朱为宏, 化学学报, 2016, 74, 351.) (c) Bian, S.; Ye, J.-H.; Fan, Z.; Zhang, W.-C.; Wang, L.-Y. Chin. J. Org. Chem. 2016, 36,855 (in Chinese). (市松, 叶家海, 樊政, 张文超, 王乐勇, 有 机化学, 2016, 36, 855.)

[21] Wang, R. B.; Yuan, W. Z.; Zhu, X. Y. Chin. J. Polym. Sci. 2015, 33, 680 .
[22] Zhao, E.; Lam, J. W. Y.; Meng, L.; Hong, Y.; Deng, H.; Bai, G.; Tang, B. Z. Macromolecules 2014, 48, 64

[23] Miao, X.; Liu, T.; Zhang, C.; Geng, X.; Meng, Y.; Li, X. Phys. Chem. Chem. Phys. 2016, 18, 4295

[24] Niu, S.; Yan, H.; Chen, Z.; Yuan, L.; Liu, T.; Liu, C. Macromol. Rapid Commun. 2016, 37, 136.

[25] Mao, B.-W.; Jia, M.; Zhang, L.; Cao, S.-K. Polym. Bull. 2014, (12), 157 (in Chinese). (毛保卫, 贾明, 张丽，曹少魁，高分子通报， 2014, (12), 157.)

(Qin, X.) 